\title{
Measuring the impact of an educational intervention in rheumatoid arthritis: An open-label, randomized trial
}

\author{
Laëtitia Michou ${ }^{1,2,3}\left(\mathbb{D}\right.$, Anne-Sophie Julien ${ }^{4}\left(\mathbb{0}\right.$, Holly O. Witteman ${ }^{5}$, Jean Légaré ${ }^{\circledR}$, Lucie Ratelle ${ }^{1}$,

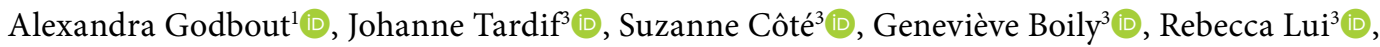

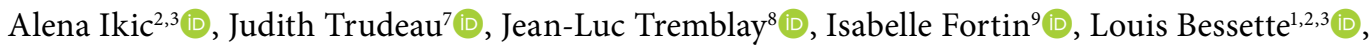 \\ Anne-Laure Chetaille ${ }^{1,2,3}$ (D), Paul R Fortin ${ }^{1,2,3} \mathbb{D}$ \\ ${ }^{1} \mathrm{CHU}$ de Québec-Université Laval Research Centre, Québec (Québec) Canada \\ ${ }^{2}$ Department of Medicine, Division of Rheumatology, Université Laval, Québec (Québec) Canada \\ ${ }^{3} \mathrm{CHU}$ de Québec-Université Laval, Québec (Québec) Canada \\ ${ }^{4}$ Department of Mathematics and statistics, Université Laval, Québec (Québec) Canada \\ ${ }^{5}$ Department of Family Medicine and Emergency Medicine, Faculty of Medicine, Université Laval, Québec (Québec) Canada \\ ${ }^{6}$ Arthritis Alliance of Canada, Québec (Québec) Canada \\ ${ }^{7}$ Department of Rheumatology, Hôtel-Dieu de Lévis, Lévis, (Québec) Canada \\ ${ }^{8}$ Clinique de Rhumatologie du Centre du Québec, Trois-Rivières, (Québec) Canada \\ ${ }^{9}$ Centre de Rhumatologie de l'Est du Québec, Rimouski, (Québec) Canada
}

\begin{abstract}
Objectives: This study aims to determine whether patients with active rheumatoid arthritis (RA), either starting on or changing biological or targeted synthetic disease-modifying antirheumatic drugs (DMARDs), demonstrate better self-management safety skills three months after receiving a multidisciplinary educational intervention compared to patients receiving usual care.

Patients and methods: Between October 2015 and October 2018, this open-label, randomized-controlled trial included a total of 107 RA patients ( 27 males, 80 females; mean age: $60.2 \pm 10.4$ years; range, 54 to 71 years) who were on treatment or in whom treatment was changed with a biological or targeted synthetic DMARD. The patients were randomized into two groups: Group 1 ( $n=57$ ) received additional intervention with educational DVD and one teleconference session and Group $2(n=55)$ received usual care and were offered the intervention at three months. All patients underwent a final visit at six months. At each visit, the patients completed the BioSecure questionnaire measuring the self-care safety skills, a behavioral intention questionnaire, and the Beliefs about Medicines Questionnaire (BMQ).

Results: No significant difference was observed in the Biosecure score at three months between the two groups ( $\mathrm{p}=0.08$ ). After pooling the first three-month data in Group 1 and the last three-month data in Group 2, the mean score of the BioSecure questionnaire increased to 7.10 \pm 0.92 in the group receiving educational intervention $(p<0.0001)$. This increase was maintained at six months in Group 2 ( $p=0.88)$. The rate of appropriate behavioral intention increased over time ( $76 \%$ at baseline and $85 \%$ at six months for both groups). There was no significant change in the BMQ $(p=0.44$ to 0.84$)$.
\end{abstract}

Conclusion: The development of an educational DVD followed by a teleconference seem to improve self-care safety skills of the patients in practical situations.

Keywords: Health behaviors, patient education, rheumatoid arthritis, questionnaire.

Received: May 07, 2021 Accepted: July 28, 2021 Published online: December 24, 2021

Correspondence: Laëtitia Michou, MD. Rhumatologie-R4774, CHU de Québec-Université Laval, 2705 boulevard Laurier, Québec, QC G1V 4G2, Canada. Tel: +1-418-654-2178 e-mail: laetitia.michou@crchudequebec.ulaval.ca

\section{Citation:}

Michou L, Julien AS, Witteman HO, Légaré J, Ratelle J, Godbout A, et al. Measuring the impact of an educational intervention in rheumatoid arthritis: An open-label, randomized trial. Arch Rheumatol 2022;37(2):169-179. 
Patient self-management is a key component in the approach to inflammatory arthritis models of care. ${ }^{1}$ Self-management requires that patients know how to manage their condition and are equipped to put that knowledge into practice by taking appropriate actions. Strategies to improve self-care knowledge and health behaviors are required to help provide high-quality care to all patients with arthritis. This information needs to be accessible to all patients, including those who face barriers such as accessing specialty care due to distance, or those who are disadvantaged by low health literacy. Patients with lower health literacy have worse outcomes across many conditions, ${ }^{2,3}$ including more severe rheumatoid arthritis (RA). ${ }^{2,3}$ Patient therapeutic education has been increasingly recognized as an integral component of chronic disease management as the part of their usual care. Recommendations for the delivery of patient education to individuals with inflammatory arthritis were published by the European League Against Rheumatism (EULAR). ${ }^{4}$ The ultimate goal of such self-management is to promote better long-term outcomes and to use healthcare resources more efficiently. ${ }^{5-7}$

It is not always possible to provide personalized therapeutic education sessions for all patients with inflammatory arthritis, particularly in remote areas of the country. A requirement to attend sessions in person is a barrier for many patients living far from university hospitals, or for those with personal obligations. Moreover, mobility limitations, lack of internet access, and unfamiliarity with technology make it difficult for some of our patients to access information about their condition. Even for those who can seek information on their own, the language barrier can cause difficulties, limiting the use of some of the available resources effectively. Adequate and reliable information on inflammatory arthritis offered in the appropriate language and adapted to the local healthcare system is scarce.

The goal of patient education in the context of a self-managed condition is to support patients with optimized self-management. This requires helping patients to not only learn information that may be new to them, but to also put this new knowledge into practice. Challenges with the latter may explain the negative results reported in published studies ${ }^{8}$ or the minor impact of patient education on disease control. ${ }^{9-12}$ The BioSecure questionnaire was developed and further validated for this purpose. ${ }^{13}$ The Theory of Planned Behavior $(\mathrm{TPB})^{14}$ is a validated theoretical framework that describes how behavior is predicted by behavioral intentions. The latter are, in turn, predicted by attitudes toward the behavior, subjective norms, and perceived behavioral control. Although this theory has not been extensively applied to arthritis, ${ }^{15,16}$ it offers a promising approach with which to structure and evaluate patient educational interventions. Targeting known determinants of behavior, not only knowledge, improves the likelihood that an educational intervention would influence self-management.

The primary objective of this study was to investigate whether patients demonstrated better self-care safety skills as assessed by the BioSecure questionnaire ${ }^{13}$ in practical situations three months after receiving a multidisciplinary educational intervention, compared to patients who received regular care. The secondary objective was to evaluate the impact of the educational intervention on behavioral intentions and beliefs and the adherence to RA medications in patients receiving the intervention, compared to those receiving usual care.

\section{PATIENTS AND METHODS}

\section{Trial design and population}

This unblinded, open-label, randomizedcontrolled trial was conducted at the $\mathrm{CHU}$ de Québec-Université Laval and Hôtel-Dieu de Lévis Hospital, Department of Rheumatology between October 2015 and October 2018. A total of 107 RA patients (27 males, 80 females; mean age: $60.2 \pm 10.4$ years; range, 54 to 71 years) were included in the study. The patients enrolled in the study had active RA and either started or changed biological or targeted synthetic diseasemodifying antirheumatic drugs (DMARDs) at the time of enrollment. Inclusion criteria were as follows: (i) age $\geq 18$ years, (ii) diagnosis of RA according to the treating rheumatologist, (iii) active RA as per rheumatologist evaluation, (iv) receiving at least two traditional conventional synthetic disease-modifying anti-rheumatic drugs (csDMARDs) in the past, and ( $v$ ) requiring the addition or change of a biological or targeted synthetic DMARD, according to their 
rheumatologist. Exclusion criteria were as follows: (i) inability to consent, (ii) inability to answer questionnaires, (iii) inability to view a DVD at home, and iv) inability to participate in a teleconference.

The patients were randomized into two groups: Group $1(n=57)$ received additional intervention with educational DVD and one teleconference session and Group $2(n=55)$ received usual care and were offered the intervention at three months (Figure 1). The patient self-care safety skills were evaluated using the BioSecure questionnaire, and the behavioral intentions and beliefs were assessed according to the medications using structured questionnaires at baseline, three, and six months. Data on RA medication adherence was obtained from pharmacy records with patient authorization; i.e., biological or targeted synthetic DMARDs and conventional synthetic diseasemodifying anti-rheumatic drugs (csDMARDs), for three months preceding enrollment and up to six months afterwards.

\section{Randomization and intervention}

The patients were randomized at a ratio of 1:1 to one of two arms, using block randomization, with blocks of 10 , stratified by sex, to account for the overrepresentation of women with RA. The randomization list was generated before the beginning of the study by a statistician using the SAS version 9.4 software (SAS Institute Inc., CA, USA).

After randomization into two groups, Group 1 received the educational DVD with the instruction to review its contents within the next two weeks. Between two and four weeks after randomization, reinforcement teleconferences were held for small groups with a maximum of 10 attendees per session for every participant who confirmed having watched the DVD. Each participant attended to only one teleconference. Usual care consisted of regular follow-up with the treating rheumatologist and the opportunity to pose questions to the rheumatology nurse by phone.

Development of the DVD: The goal of the 40-min DVD was to provide patients information about RA and its management. It includes 12 sections: (i) general information on arthritis, (ii) information on treatments (efficacy, side effects and disease control), (iii) vaccination, childbearing, surgery, (iv) how

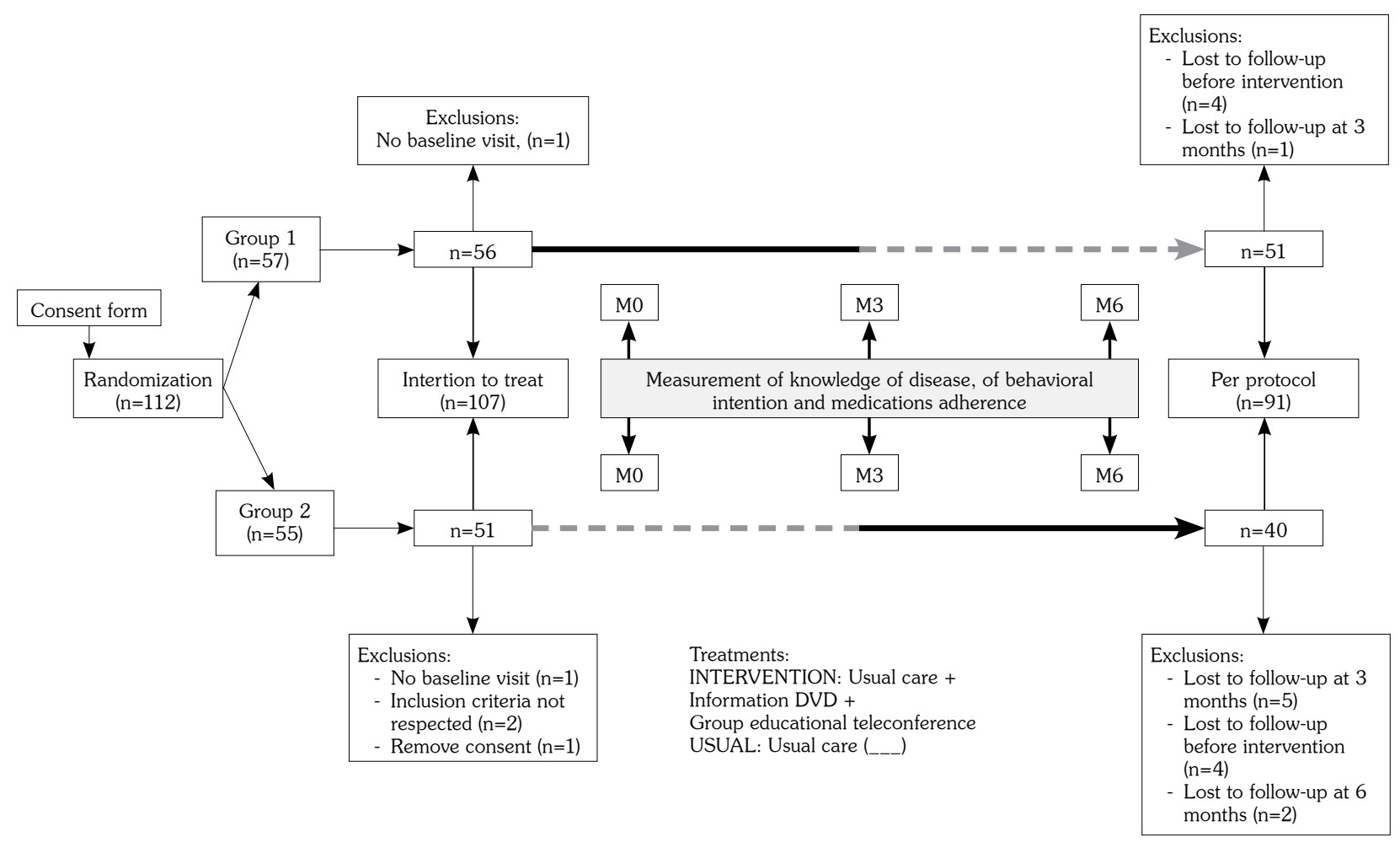

Figure 1. Study flow chart. 
to inject subcutaneous medications, $(v)$ advances in research, future medications and genetics, (vi) fatigue, depression and pain, (vii) how occupational therapy, including joint protection techniques, and orthotics can contribute to the management of arthritis, (viii) concepts of physiotherapy, including different categories of exercise and advice on how to achieve success (with regard to fatigue or pain), (ix) nutrition and arthritis, $(x)$ services in the community including offerings by the Arthritis Society, (xi) cost of treatment, information on the healthcare system and drug reimbursement strategies in the province, and (xii) a patient testimonial.

As low health literacy has been reported as a key limitation in educational interventions in patients with arthritis, ${ }^{17}$ we ensured that the DVD was suitable for this subpopulation. A plain language targeting a Grade 6 reading level was used. Information was presented clearly and simply, with the introduction of one new concept at a time, and repetition of key points. After completion of a preliminary version, we consulted five knowledgeable arthritis patients to determine whether the DVD was acceptable for this patient audience. Their comments and suggestions were incorporated into the final version. In addition to the DVD, the participants were sent a memory aid, outlining the important information from the DVD.

Group educational teleconference: In parallel with the development of the DVD, a teleconference was designed with the aim of helping patients to increase their self-efficacy (confidence in their ability to overcome obstacles) for self-management. The teleconferences were designed to review the key points from the 12 chapters of the DVD and were hosted by a rheumatologist, research assistant, physiotherapist, occupational therapist, and a rheumatology nurse from our team. Brief summary presentations by participating health professionals were followed by an open forum to answer questions from participants and to discuss self-management strategies.

\section{Outcome measurements}

The BioSecure questionnaire was used to assess patients' self-care safety skills knowledge. ${ }^{13}$ This 55-item questionnaire measures the self-care safety skills of patients treated with a biological agent through the use of both knowledge and coping strategies. This questionnaire primarily contains true-or-false questions to assess the patient's knowledge. Each correct answer is awarded one point. The total is, then, transformed into a percentage. A value of $100 \%$ represents a patient with a perfect knowledge. After referring to published data on the TPB,${ }^{14}$ we developed a questionnaire to evaluate behavioral intention, focusing on the appropriate or inappropriate intention to administer an injection of a biological agent while presenting symptoms of infection. This is comprised of 12 questions to be answered using a seven-point Likert scale, from "Completely disagree" to "Completely agree". Higher values correspond to good attitude, norm, control, or intention. The questionnaire includes two questions on attitude, six questions on perceived norms, two questions on perceived behavioral control, one question on intention, and one question on past behavior. Intention was dichotomized for analysis purposes, with values 1 to 3 considered to be health-promoting intentions, while values 4 to 7 were healthdamaging intentions. For medication beliefs, we adapted the published Belief about Medicines Questionnaire (BMQ) to RA medication. ${ }^{18,19}$ This questionnaire is comprised of 18 questions to evaluate the four subscales of the $\mathrm{BMQ}$; i.e., specific-necessity (five questions), specific-concern (five questions), general-harm (four questions), and general-overuse (four questions), and was completed by making one selection from among five choices ranging from "completely agree" to "completely disagree". The score of each subscale is the sum of its items, and as such has a maximum value of 25 or 20 points depending on the associated number of items. A high value corresponds to greater necessity, concern, harm, or overuse. None of these questionnaires were under license. Medication adherence was assessed using pharmacy records data. The proportion of adherence was calculated by dividing the quantity received by the quantity prescribed for each month starting three months before enrollment until six months after. Biological or targeted synthetic DMARDs and csDMARDs were treated separately. The mean proportion of adherence per medication was calculated for each time period (three months before the study to baseline, baseline to three months, and three months to 
six months) by taking the average of all months included in each of the three periods. For each of these periods, the patient was considered adherent, if the mean value was equal to $100 \%$. However, sensitivity analyses for adherence were also conducted using a threshold greater or equal to $80 \%$, as used in most clinical studies. ${ }^{20-23}$

\section{Statistical analysis}

The study power and sample size calculation were performed using the SAS version 9.3 software (SAS Institute Inc., CA, USA). Accordingly, the sample size was calculated to detect a medium difference (Cohen's $d=0.50$ ) between the groups at three months using a Student t-test with 95\% confidence interval (CI) and 80\% study power. Considering a dropout rate of $10 \%$, a sample size of 128 was increased to 142 patients, with 71 in each group.

Statistical analysis was performed using the SAS version 9.4 software (SAS Institute Inc., CA, USA). Descriptive data were expressed in mean \pm standard deviation, median (min-max) or number and frequency, where applicable. The Cronbach's alpha coefficients were calculated for each subscale of the behavioral questionnaire and the BMQ at baseline to assess internal consistency. The Wilcoxon tests for independent samples were performed to test whether the distribution of quantitative variables at baseline differed between patients with complete versus incomplete follow-ups, while an exact chi-square test was used for qualitative data. Graphs were generated to show the evolution of the mean of continuous scores with standard deviation over time, for each group. Both intention-totreat and per protocol analyses were performed. Repeated-measures analyses of variance (RM-ANOVA) estimated using a linear mixed model were performed to analyze the effect of visit, group, and their interaction on each of the continuous scores. The Kenward-Rogers degrees of freedom were considered. Repeatedmeasures logistic regression estimated using a generalized linear mixed model was used to test the same effects on the behavioral intention ("health-promoting" or "health-damaging"), as well as on medication adherence. This analysis on adherence for biological or targeted synthetic DMARDs only included patients who switched from another biological or targeted synthetic DMARD, as those who began their first biological treatment at enrollment did not have measurements of adherence prior to the study, precluding testing a pre-post difference. A $p$ value of $<0.05$ was considered statistically significant.

\section{RESULTS}

\section{Description of study participants}

Of a total of 112 active RA patients randomized to this study, 107 were included in the intention-to-treat analyses and five patients were excluded. A total of 91 patients completed all steps of the study. The mean disease duration was $8.6 \pm 10.6$ (range, 1 to 16 ) years at baseline (Table 1). No comparisons between the groups at baseline were performed in accordance with the CONSORT guidelines. ${ }^{24}$ Forty patients started on their first biological or targeted synthetic DMARD at baseline. The participation rate at six months was higher in the early intervention group versus the usual care $(91 \%$ vs. $78 \%$, respectively). The patients who did not complete all steps of the study had lower mean scores on the BioSecure questionnaire at baseline $(61 \pm 14$ vs. $71 \pm 13$, respectively; $Z=-3.39$, $p=0.0007)$. Furthermore, they predominantly belonged to the usual care group who received the DVD and the teleconference at three months $\left(77 \%\right.$ vs. $44 \%$, respectively; $\chi^{2}=9.09, \quad \mathrm{df}=1$, $\mathrm{p}=0.003)$, a higher proportion worked full-time (41\% vs. 30\%, respectively), and they were less often retired ( $23 \%$ vs. 49\%, respectively; $\chi^{2}=13.35, \mathrm{df}=5, \mathrm{p}=0.02$ ).

\section{Impact of educational intervention on self-care safety skills as measured by the BioSecure questionnaire in intention-to-treat analyses}

We observed a significant interaction $(\mathrm{F}=6.17, \mathrm{df}=[2,95.3], \mathrm{p}=0.0030)$ in the temporal evolution between the two groups (Figure 2), indicating that the slopes of the groups were not parallel; i.e., the groups did not improve in the same manner. After combining the two groups, we observed that the educational intervention significantly increased $(t=7.69, \mathrm{df}=175.5, \mathrm{p}<0.0001)$ the mean score by $7.1 \pm 0.9$. However, no significant differences were observed between the two groups 
Table 1. Sociodemographic and clinical characteristics of study population

\begin{tabular}{|c|c|c|c|c|c|c|}
\hline \multirow[b]{2}{*}{ Sociodemographic or clinical characteristics } & \multicolumn{3}{|c|}{ Usual care $(\mathrm{n}=51)$} & \multicolumn{3}{|c|}{ Intervention $(n=56)$} \\
\hline & $\mathrm{n}$ & $\%$ & Mean \pm SD & $\mathrm{n}$ & $\%$ & Mean \pm SD \\
\hline Age (year) & & & $59.2 \pm 11.4$ & & & $61.0 \pm 9.4$ \\
\hline Disease duration (year) & & & $9.2 \pm 13.3$ & & & $8.0 \pm 7.5$ \\
\hline $\begin{array}{l}\text { Sex } \\
\text { Female }\end{array}$ & 37 & 73 & & 43 & 77 & \\
\hline $\begin{array}{l}\text { Number of previous biologic DMARDs } \\
0 \\
1 \\
2 \\
3 \\
4-6 \\
14\end{array}$ & $\begin{array}{c}18 \\
11 \\
8 \\
6 \\
7 \\
1\end{array}$ & $\begin{array}{c}35 \\
22 \\
16 \\
12 \\
14 \\
2\end{array}$ & & $\begin{array}{c}21 \\
15 \\
5 \\
8 \\
6 \\
0\end{array}$ & $\begin{array}{c}38 \\
27 \\
9 \\
15 \\
11 \\
0\end{array}$ & \\
\hline $\begin{array}{l}\text { Duration of the most recent biologic DMARDs, mean in years } \pm \text { SD } \\
\text { (among } 34 \text { and } 33 \text { participants who were not bio-naïve, respectively) }\end{array}$ & & & $1.8 \pm 2.7$ & & & $2.00 \pm 2.0$ \\
\hline $\begin{array}{l}\text { Marital status } \\
\text { Domestic partnership } \\
\text { Single } \\
\text { Divorced } \\
\text { Married } \\
\text { Separated } \\
\text { Widowed }\end{array}$ & $\begin{array}{c}7 \\
8 \\
10 \\
23 \\
2 \\
1\end{array}$ & $\begin{array}{c}14 \\
16 \\
20 \\
45 \\
4 \\
2\end{array}$ & & $\begin{array}{c}12 \\
3 \\
13 \\
21 \\
1 \\
6\end{array}$ & $\begin{array}{c}21 \\
5 \\
23 \\
38 \\
2 \\
11\end{array}$ & \\
\hline $\begin{array}{l}\text { Occupation } \\
\text { Employed or working full time } \\
\text { Employed part time } \\
\text { At home } \\
\text { Disabled } \\
\text { Retired } \\
\text { Student } \\
\text { Unemployed }\end{array}$ & $\begin{array}{c}13 \\
3 \\
1 \\
8 \\
23 \\
1 \\
2\end{array}$ & $\begin{array}{l}25 \\
6 \\
2 \\
16 \\
45 \\
2 \\
4\end{array}$ & & $\begin{array}{c}19 \\
5 \\
2 \\
4 \\
24 \\
0 \\
2\end{array}$ & $\begin{array}{c}34 \\
9 \\
4 \\
7 \\
43 \\
0 \\
4\end{array}$ & \\
\hline $\begin{array}{l}\text { Current biologic or targeted synthetic DMARDs } \\
\text { Abatacept } \\
\text { Adalimumab } \\
\text { Certolizumab } \\
\text { Etanercept } \\
\text { Golimumab } \\
\text { Rituximab } \\
\text { Sarilumab } \\
\text { Tocilizumab } \\
\text { Tofacitinib }\end{array}$ & $\begin{array}{c}6 \\
7 \\
4 \\
7 \\
5 \\
4 \\
1 \\
6 \\
11\end{array}$ & $\begin{array}{c}12 \\
14 \\
8 \\
14 \\
10 \\
8 \\
2 \\
12 \\
22\end{array}$ & & $\begin{array}{l}10 \\
6 \\
5 \\
13 \\
5 \\
4 \\
1 \\
7 \\
5\end{array}$ & $\begin{array}{l}18 \\
11 \\
9 \\
23 \\
9 \\
7 \\
2 \\
13 \\
9\end{array}$ & \\
\hline
\end{tabular}

at three months $(t=1.74, d f=100.9, p=0.08)$. This effect remained stable between three and six months for the intervention group $(\mathrm{t}=0.15, \mathrm{df}=89.64$, $p=0.88$ ). Per protocol analysis yielded similar results. In the subgroup of participants starting on a first biological or targeted synthetic DMARD, a post-hoc analysis showed that the score on the BioSecure questionnaire was higher at three months in the group who received the intervention at baseline versus usual care (76 vs. 66, respectively; $\mathrm{t}=2.76, \mathrm{df}=36.44, \mathrm{p}=0.009$ ).

\section{Impact of the educational intervention on behavioral intention according to the theory of planned behavior}

In this questionnaire on behavioral intention, the internal consistency measured by Cronbach's alpha was acceptable for perceived norm (0.78), poor for attitude (0.52) and very weak for perceived behavioral control (0.18). Although the proportion of health-promoting intention appeared to increase slightly over time in intention to treat $(76 \%$ at baseline and $85 \%$ at six months for 


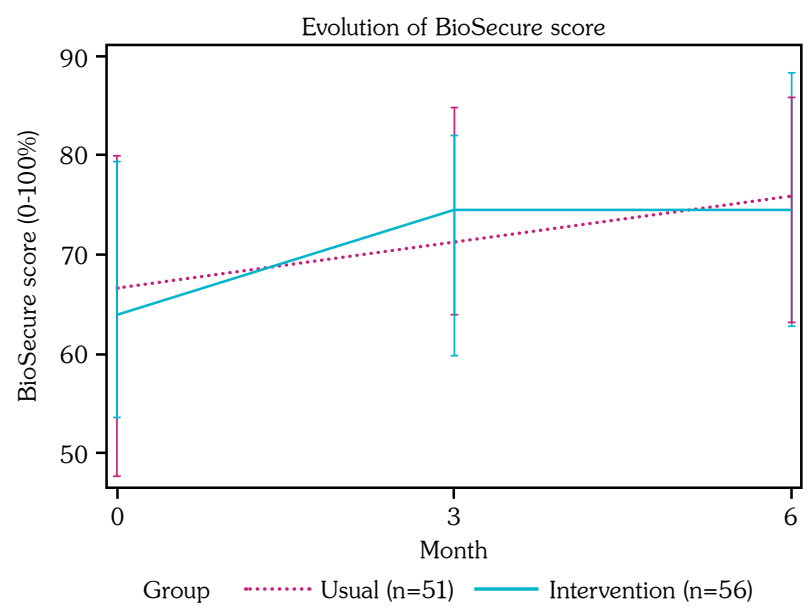

Figure 2. Impact of the educational intervention on the BioSecure score over time.

(a)

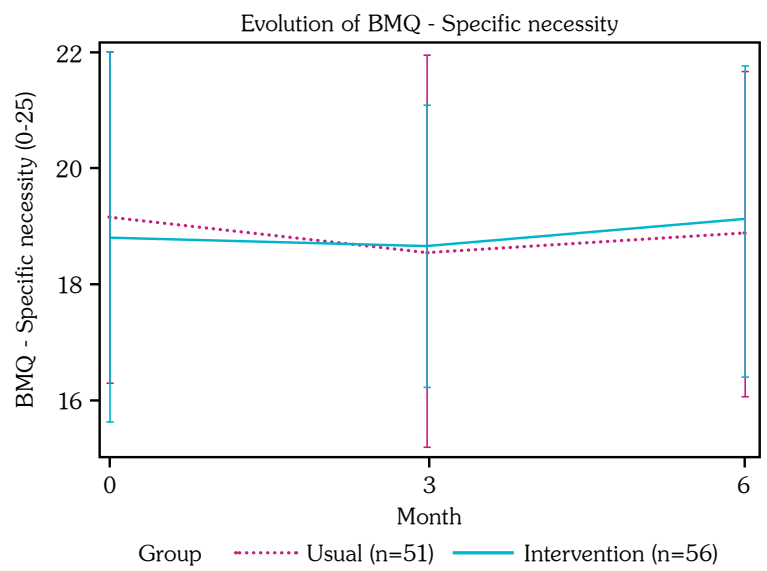

(c)

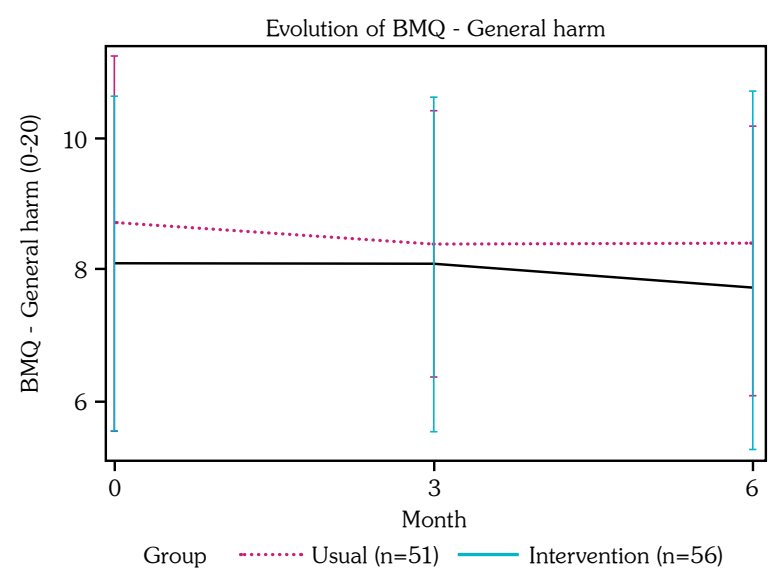

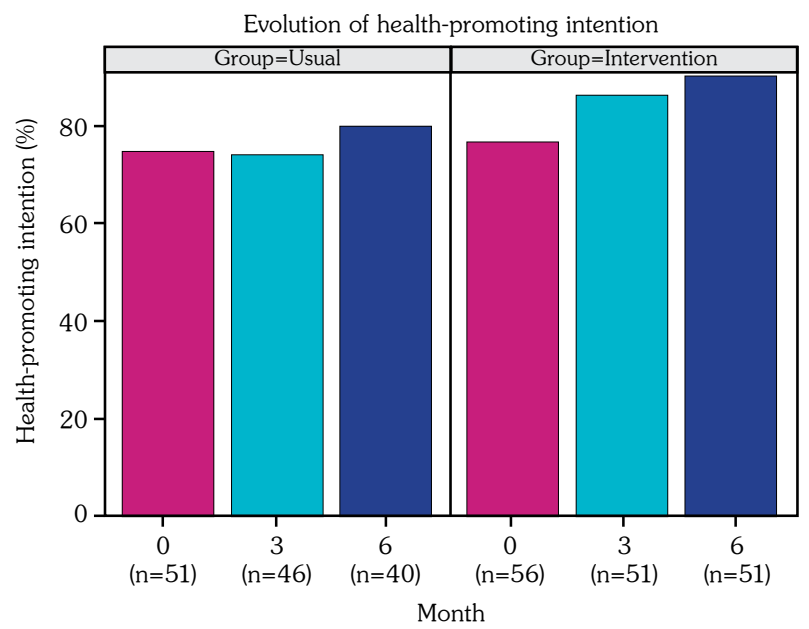

Figure 3. Impact of the educational intervention on behavioral intention over time.

(b)

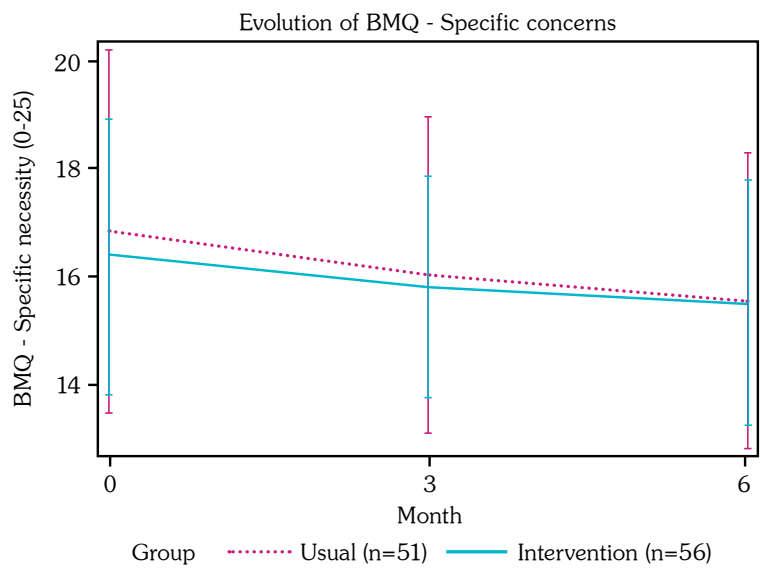

(d)

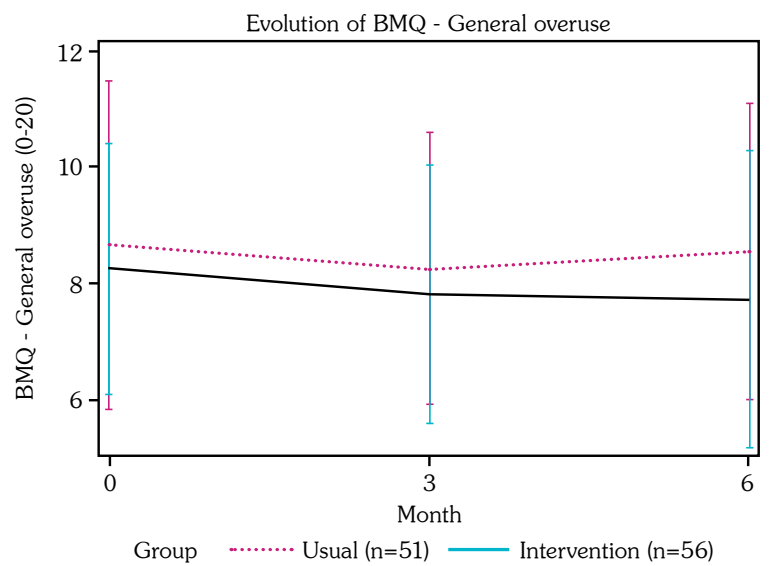

Figure 4. Impact of educational intervention on the four subscales of the beliefs about medicines questionnaire (BMQ): (a) Specific-necessity. (b) Specific-concerns. (c) General-harm. (d) General-overuse.

BMQ: Beliefs about Medicines Questionnaire. 
(a)

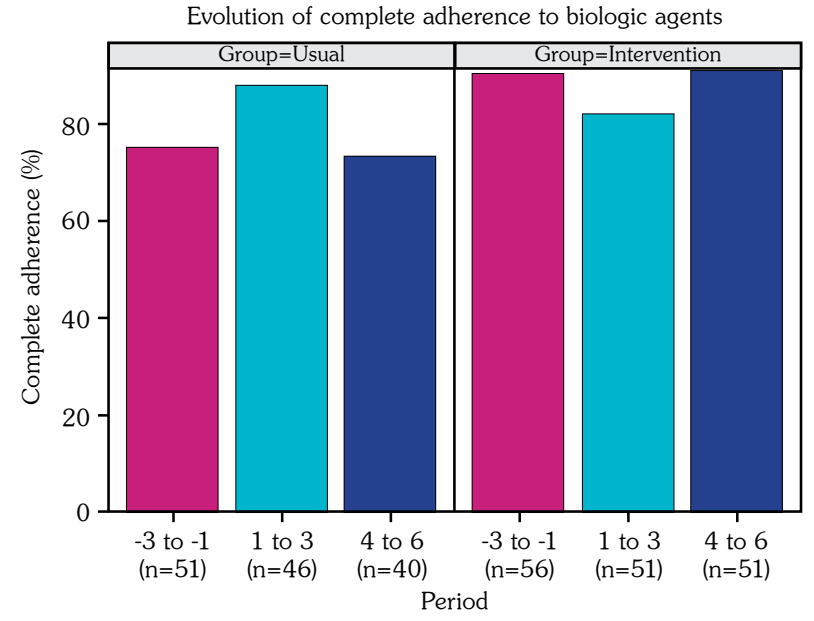

(b)

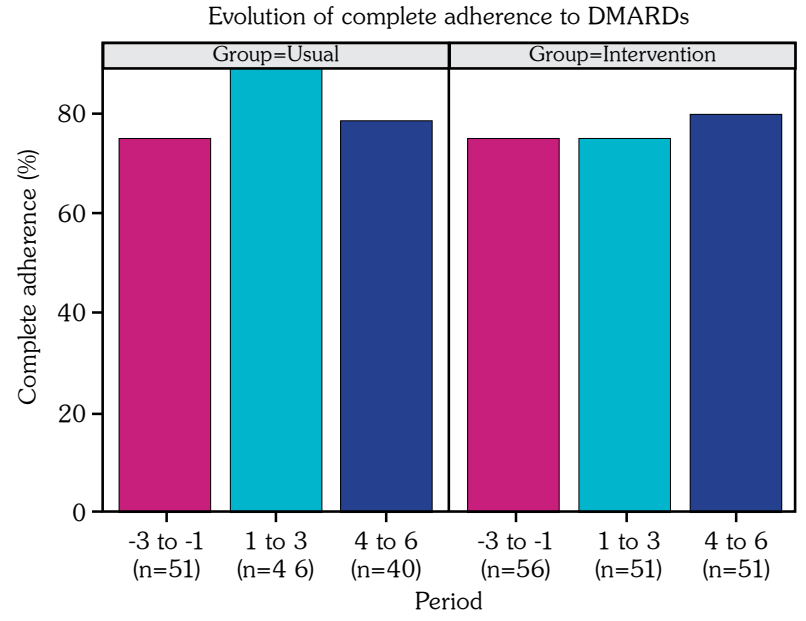

Figure 5. Impact of the educational intervention on rheumatoid arthritis medication adherence over time: (a) Biological or targeted synthetic DMARDs and (b) Conventional synthetic DMARDs.

DMARDs: Disease-modifying antirheumatic drugs.

both groups), no statistically significant differences were detected (Figure 3). Similar observations were made in the per protocol analysis.

\section{Impact of the educational intervention on rheumatoid arthritis medication beliefs and adherence}

Using the $\mathrm{BMQ}$, the internal consistency measured by Cronbach's alpha was good for the general-harm (0.73) and general-overuse (0.74) subscales, but only fair for the specificnecessity (0.43) and specific-concern (0.49) subscales. No statistically significant differences were detected for the specific necessity and general harm subscales of the BMQ. A visit effect was observed for the specific concerns and general overuse subscales $(\mathrm{F}=0.0005, \mathrm{df}=(2,93)$, $\mathrm{p}=0.0005$ and $\mathrm{F}=0.03, \mathrm{df}=(2,92.7), \mathrm{p}=0.03$, respectively), with both groups showing a similar decrease (Figure 4). Comparison of adherence to the RA medication three-to-one months before baseline versus the two-to-three-month intervention periods revealed no significant difference in adherence rates among the three periods for the two study groups $(\mathrm{F}=0.02$, $\mathrm{df}=(2,60), \mathrm{p}=0.98)$. This was also applicable to the csDMARDs $(\mathrm{F}=1.41, \mathrm{df}=(2,67), \mathrm{p}=0.25)$ (Figure 5). By applying a threshold of $\geq 80 \%$, a significant interaction $(\mathrm{F}=3.72, \mathrm{df}=(2,60)$, $\mathrm{p}=0.03$ ) was found for biological and targeted synthetic DMARDs. Multiple comparisons showed that the proportion of adherent patients was significantly higher during the final period (four to six months, $\mathrm{F}=4.06, \mathrm{df}=(1,60), \mathrm{p}=0.049$ ) for the intervention group compared to the usual care group $(94 \pm 4 \%$ vs. $73 \pm 9 \%)$. No significant effects were found with the 80\%-threshold with regard to the csDMARDs.

\section{DISCUSSION}

In this study, we investigated whether an educational intervention improved self-care safety skills, behavioral intentions, and medication beliefs and adherence among patients with RA. After pooling both arms, the intervention gave rise to an increased mean score of $7.10 \pm 0.92$ on the BioSecure questionnaire $(\mathrm{t}=7.69, \mathrm{df}=175.5$, $\mathrm{p}<0.0001$ ), with no significant difference at three months. No significant decrease was found for the educational intervention at six months in the early intervention arm (Group 1, $\mathrm{t}=0.15, \mathrm{df}=89.64$, $\mathrm{p}=0.88$ ). The proportion of appropriate intention increased at six months in both groups $(85 \pm 4 \%$ vs. $76 \pm 4 \%$, respectively at baseline), although the result did not reach statistical significance. We did not observe any change in the $\mathrm{BMQ}$ subscales between the groups or during patient follow-up. Patients with incomplete follow-up and lower scores on the BioSecure questionnaire 
were predominantly from the usual care group and were not retired. The identification of this subgroup with compromised knowledge would allow the personalization of future educational interventions. Our educational intervention approach can be adapted to motivate and engage these patients. Face-to-face counselling with a clinical nurse specialized in rheumatology and/or a closer follow-up with a rheumatologist should be considered for these patients, who are less likely to self-manage their arthritis.

In our study, at baseline, the mean BioSecure score was 63.9 in Group 2 and 66.6 in Group 1. This score increased to 74 in both groups at six months, which is very similar to the median scores of 72 and 73 reported in a survey of 677 patients in France, ${ }^{25,26}$ although access to patient education and the health literacy levels are quite different between both countries. The absence of correlation between the improved patient knowledge and intention/belief has already been published. Better evaluation tools have to be designed for a more accurate assessment of patient education, and caregiver involvement in patient education is of paramount importance. ${ }^{27}$ Behavioral intention was evaluated using a questionnaire derived from the TPB, which was reported to be of moderate support in $\mathrm{RA} .{ }^{15}$ In future projects, the Patient Activation Measure, a 22-item questionnaire to measure knowledge, skill and confidence for self-management, may be more appropriate. ${ }^{28}$ In the present study, we found no association between the subscales of the BMQ and adherence to DMARDs. There are no reports in the literature regarding the association between the BMQ and adherence to biologics, whereas the necessity score is independently associated with adherence to oral csDMARDs. ${ }^{29}$ This observation was confirmed in other studies, reporting that lower necessity beliefs were associated with medication non-adherence, ${ }^{30}$ as well as higher concerns about taking medication. ${ }^{31}$ The variation in biological agent adherence with time during our study is unclear, since the result differed depending on the adherence threshold used. A significant difference was only observed between groups in the third period in the model with 100\% adherence. This difference might be related to a non-significant decrease in adherence after receiving the intervention (three months for the intervention group, six months for the usual care group). An increase was observed for the intervention group at six months. A decrease in adherence to RA medication immediately following the intervention may be explained by the participants' fear of the medication side effects presented in the educational intervention. The increase in RA medication adherence afterwards can be attributed to the recurrence of RA symptoms and/or reassurance from the treating rheumatologist and other healthcare providers.

This study has certain limitations. Although we used, or adapted from the literature, validated questionnaires to assess patients' self-management knowledge, behavioral intention, and medication beliefs, the behavioral intention questionnaire was not validated prior to launching the study. Intention was measured with only one question: therefore, it was not possible to evaluate its internal consistency. Other scales (attitude and control) had an insufficient internal consistency. The internal consistency for two subscales was unsatisfactory for the medication beliefs scale in the adapted version. Validation of these scales should be performed prior to their use in future studies.

Due to low recruitment, the smaller sample size of 107 patients (91 of whom completed all steps of the study), instead of the anticipated 128 , may have resulted in a lack of power to detect significant and clinically important changes. Additional information from patient support programs was available to all participants while starting a new biological or targeted synthetic DMARD that may have also contributed to the absence of a statistically significant difference between the intervention and usual care groups at three months. The inference of the models, either in intention-to-treat or per protocol, was limited by the distinct characteristics between the participants lost to follow-up and those who completed the study. Moreover, the short timeline of this study and the limited followup period of six months prevented us from assessing whether our intervention would lead to better disease self-management in the mid- to long-term.

The RA medication adherence analysis is also subject to certain limitations. We were only able to perform the analysis on the subgroup of 
participants for whom we had adherence data from before and during the study; i.e., those who completed the study. Thus, further studies on other populations and in other contexts, in particular early arthritis management, would be of interest.

Nonetheless, as this study showed, videos represent an effective means of delivering information to patients with RA as a means to improve their self-care safety skills. We believe that these videos would be available online for accurate self-management information. Our results would also allow the development of other innovative research projects on patient education, such as a passport for patients with RA or an interactive navigator using the same design as that developed for systemic lupus erythematosus. ${ }^{32}$

In conclusion, the development of an innovative educational intervention consisting of a DVD followed by a teleconference can improve the self-care safety skills of patients in practical cases over a six-month period. However, it seems that our educational intervention has no significant impact on behavioral intentions or medication beliefs up to six months.

Ethics Committee Approval: The study protocol was approved by the CHU de Québec-Université Laval Ethics Committee (MP-20-2015-2242). The study was conducted in accordance with the principles of the Declaration of Helsinki.

Patient Consent for Publication: A written informed consent was obtained from each patient.

Data Sharing Statement: The data that support the findings of this study are available from the corresponding author upon reasonable request.

Author Contributions: Study design: LM, HOW, PRF. Patient recruitment and acquisition of data: LM, LR, AG, JT, SC, GB, RL, AI, JT, JLT, IF, LB, ALC, PRF. Analysis and interpretation of data: ASJ, LM, HOW, JL, JT, SC, GB, RL, AI, JT, JLT, IF, LB, ALC, PRF. Revision of manuscript content: all authors. Approving final version of manuscript: all authors. LM takes responsibility for the integrity of the data analysis.

Acknowledgments: We would like to thank $\mathrm{Dr}$ Laure Gossec and Dr Catherine Beauvais from the French Rheumatology Society for helpful discussions on the BioSecure questionnaire and the $\mathrm{CHU}$ de QuébecUniversite Laval hospital for allowing the participation of healthcare professionals to our research project. We also would like to thank Mrs Muriel Kelly for the manuscript revision.
Conflict of Interest: The authors declared no conflicts of interest with respect to the authorship and/or publication of this article.

Funding: Dr. Michou is supported by a career award from the Fonds de recherche du Québec-Santé. HOW is Dr Witteman supported by a Tier 2 Canada Research Chair in Human-Centred Digital Health. Dr. Fortin is supported by a Tier 1 Canada Research Chair on Systemic Autoimmune Rheumatic Diseases. This study was funded by a CIORA grant from the Canadian Rheumatology Association and by The Arthritis Society through Rheumatic Health Unit fundings.

\section{REFERENCES}

1. Arthritis Alliance of Canada. Joint Action on Arthritis: A Framework to Improve Arthritis Prevention and Care in Canada. Available at: wwwarthritisallianceca 2012.

2. Hirsh JM, Boyle DJ, Collier DH, Oxenfeld AJ, Nash A, Quinzanos I, et al. Limited health literacy is a common finding in a public health hospital's rheumatology clinic and is predictive of disease severity. J Clin Rheumatol 2011;17:236-41.

3. Baker DW, Wolf MS, Feinglass J, Thompson JA, Gazmararian JA, Huang J. Health literacy and mortality among elderly persons. Arch Intern Med 2007;167:1503-9.

4. Zangi HA, Ndosi M, Adams J, Andersen L, Bode $C$, Boström $C$, et al. EULAR recommendations for patient education for people with inflammatory arthritis. Ann Rheum Dis 2015;74:954-62.

5. Stockl KM, Shin JS, Lew HC, Zakharyan A, Harada AS, Solow BK, et al. Outcomes of a rheumatoid arthritis disease therapy management program focusing on medication adherence. J Manag Care Pharm 2010;16:593-604.

6. Nour K, Laforest S, Gauvin L, Gignac M. Behavior change following a self-management intervention for housebound older adults with arthritis: An experimental study. Int $\mathrm{J}$ Behav Nutr Phys Act 2006;3:12.

7. Brady TJ. Cost implications of self-management education intervention programmes in arthritis. Best Pract Res Clin Rheumatol 2012;26:611-25.

8. Riemsma RP, Kirwan JR, Taal E, Rasker JJ. Patient education for adults with rheumatoid arthritis. Cochrane Database Syst Rev 2003;(2):CD003688.

9. Riemsma RP, Taal E, Rasker JJ. Group education for patients with rheumatoid arthritis and their partners. Arthritis Rheum 2003;49:556-66.

10. Riemsma RP, Taal E, Kirwan JR, Rasker JJ. Systematic review of rheumatoid arthritis patient education. Arthritis Rheum 2004:51:1045-59.

11. Foster G, Taylor SJ, Eldridge SE, Ramsay J, Griffiths CJ. Self-management education programmes by lay 
leaders for people with chronic conditions. Cochrane Database Syst Rev 2007;(4):CD005108.

12. Giraudet-Le Quintrec JS, Mayoux-Benhamou A, Ravaud P, Champion K, Dernis E, Zerkak D, et al. Effect of a collective educational program for patients with rheumatoid arthritis: A prospective 12-month randomized controlled trial. J Rheumatol 2007;34:1684-91.

13. Gossec L, Fautrel B, Flipon É, Lecoq d'André F, Marguerie L, Nataf $\mathrm{H}$, et al. Safety of biologics: Elaboration and validation of a questionnaire assessing patients' self-care safety skills: The BioSecure questionnaire. An initiative of the French Rheumatology Society Therapeutic Education section. Joint Bone Spine 2013;80:471-6.

14. Ajzen I. The theory of planned behavior. Organizational Behavior and Human Decision Processes 1991;50:179-211.

15. Strating MM, van Schuur WH, Suurmeijer TP. Contribution of partner support in self-management of rheumatoid arthritis patients. An application of the theory of planned behavior. J Behav Med 2006;29:51-60.

16. John H, Hale ED, Bennett P, Treharne GJ, Carroll D, Kitas GD. Translating patient education theory into practice: Developing material to address the cardiovascular education needs of people with rheumatoid arthritis. Patient Educ Couns 2011;84:123-7.

17. Rhee RL, Von Feldt JM, Schumacher HR, Merkel PA. Readability and suitability assessment of patient education materials in rheumatic diseases. Arthritis Care Res (Hoboken) 2013;65:1702-6.

18. Horne R, Weinman J, Hankins M. The beliefs about medicines questionnaire: The development and evaluation of a new method for assessing the cognitive representation of medication. Psychology \& Health 1999; 14:1-24.

19. Fall E, Gauchet A, Izaute M, Horne R, Chakroun N. Validation of the French version of the Beliefs about Medicines Questionnaire (BMQ) among diabetes and HIV patients. European Review of Applied Psychology 2014;64:335-43.

20. Haynes RB, Taylor DW, Sackett DL, Gibson ES, Bernholz CD, Mukherjee J. Can simple clinical measurements detect patient noncompliance? Hypertension 1980;2:757-64.

21. Caro JJ, Ishak KJ, Huybrechts KF, Raggio G, Naujoks C. The impact of compliance with osteoporosis therapy on fracture rates in actual practice. Osteoporos Int 2004;15:1003-8.

22. Doró P, Benko R, Kosik E, Matuz M, Tóth K, Soós G. Utilization of oral antihyperglycemic drugs over a 7-year period (1998-2004) in a Hungarian population and adherence to drug therapy. Eur J Clin Pharmacol 2005;61:893-7.

23. Hansen RA, Farley JF, Droege M, Maciejewski ML. A retrospective cohort study of economic outcomes and adherence to monotherapy with metformin, pioglitazone, or a sulfonylurea among patients with type 2 diabetes mellitus in the United States from 2003 to 2005. Clin Ther 2010;32:1308-19.

24. Moher D, Hopewell S, Schulz KF, Montori V, Gøtzsche PC, Devereaux PJ, et al. CONSORT 2010 explanation and elaboration: Updated guidelines for reporting parallel group randomised trials. Int J Surg 2012;10:28-55.

25. Rat AC, Fautrel B, Flipon E, Gossec L, Marguerie $\mathrm{L}$, Nataf $\mathrm{H}$, et al. Factors associated with knowledge and safety skills of arthritis patients receiving biologics: A survey of 677 patients. Joint Bone Spine 2017;84:163-8.

26. Orefice D, Beauvais C, Gossec L, Flipon E, Fautrel $\mathrm{B}$, Marguerie L, et al. Cross-sectional study of self-care safety skills in 677 patients on biodrugs for inflammatory joint disease. Joint Bone Spine 2014;81:502-7.

27. Giraudet JS. Therapeutic education of patients and relatives. Rev Prat 2018;68:819-25. French.

28. Hibbard JH, Stockard J, Mahoney ER, Tusler M. Development of the Patient Activation Measure (PAM): Conceptualizing and measuring activation in patients and consumers. Health Serv Res 2004;39:1005-26.

29. McCulley C, Katz P, Trupin L, Yelin EH, Barton JL. Association of medication beliefs, self-efficacy, and adherence in a diverse cohort of adults with rheumatoid arthritis. J Rheumatol 2018;45:163642.

30. Zwikker HE, van Dulmen S, den Broeder AA, van den Bemt $\mathrm{BJ}$, van den Ende $\mathrm{CH}$. Perceived need to take medication is associated with medication non-adherence in patients with rheumatoid arthritis. Patient Prefer Adherence 2014;8:1635-45.

31. Cea-Calvo L, Marín-Jiménez I, de Toro J, FusterRuizdeApodaca MJ, Fernández G, Sánchez-Vega $\mathrm{N}$, et al. Association between non-adherence behaviors, patients' experience with healthcare and beliefs in medications: A survey of patients with different chronic conditions. Curr Med Res Opin 2020;36:293-300.

32. Neville C, Da Costa D, Rochon M, Peschken CA, Pineau CA, Bernatsky $\mathrm{S}$, et al. Development of the lupus interactive navigator as an empowering webbased eHealth tool to facilitate lupus management: Users perspectives on usability and acceptability. JMIR Res Protoc 2016;5:e44. 